\title{
HIV-negative Rifampicin Resistance/Multidrug- Resistant Extrapulmonary Tuberculosis in China from 2015 to 2019: A clinical retrospective investigation study from a National Tuberculosis Clinical Research Center
}

Jun Ma

Tongji University Affiliated Shanghai Pulmonary Hospital

Hongcheng Liu

Tongji University Affiliated Shanghai Pulmonary Hospital

Jingjing Wang

Tongji University Affiliated Shanghai Pulmonary Hospital

Wenting Li

Tongji University Affiliated Shanghai Pulmonary Hospital

Lin Fan

Tongji University Affiliated Shanghai Pulmonary Hospital

Wenwen Sun ( $\nabla$ sunwenwen_1583@163.com )

Tongji University Affiliated Shanghai Pulmonary Hospital https://orcid.org/0000-0003-3465-5235

\section{Research Article}

Keywords: Rifampicin Resistance, Multidrug Resistant, Extrapulmonary tuberculosis, Clinical characteristics, Xpert MTB/RIF, Fluoroquinolones

Posted Date: February 7th, 2022

DOI: https://doi.org/10.21203/rs.3.rs-636342/v2

License: (a) (1) This work is licensed under a Creative Commons Attribution 4.0 International License. Read Full License 


\section{Abstract}

Background: China is the region with a high global burden of Rifampicin Resistance \Multidrug-Resistant tuberculosis (RR/MDR-TB) and low HIV incidence. Our aim was to assess the clinical and demographic characteristics of RR/MDR - extrapulmonary tuberculosis (EPTB) from 2015 to 2019, to provide evidence for the prevention and control of the disease in high TB burden areas.

Methods: We investigated the clinical and demographic data of all MDR/ RR -EPTB cases in a TB specialized hospital from China and compared the cases with the MDR/ RR-pulmonary tuberculosis (PTB) patients over the same period.

Results: Of the RR/MDR-TB patients enrolled 15.4 were EPTB. The most common anatomical site was pleural/chest wall (25.9\%). Compared with RR囚MDR-PTB囚females were more likely to be susceptible to RR/MDR-EPTB (OR 1.65, 95\% Cl 1.52-1.77) \ the risk of RR/MDR-EPTB forヌ25-44『years group increased (OR 1.61, 95\% Cl 1.52-1.77), and then decreased with the increasing age (OR1.48 95\% Cl 0.74-1.69 for 4465 years group and OR $2.2395 \% \mathrm{Cl} 0.98-2.71$ for $\geq 65$ years group) $\nabla$ more likely to be newly diagnosed $₫ p$ $<0.01$ \and less likely to to combine with diabetes $₫ P<0.01$ ) $\nabla$ more dependent on GeneXpert MTB $\triangle \mathrm{RIF}$ $\triangle X$ pert $\triangle 90.9 \%$ ) for diagnosis $\square$ with significantly higher rates of pre-XDR $\square X D R$ and significantly lower favorable treatment outcomes $₫$ both $p<0.01 \rrbracket$.

Conclusion: There are clinical and demographic differences between RR/MDR-PTB and RR/MDR-PTB. Xper should be recommended at an early stage for suspected patients囚and fluoroquinolones should be used cautiously for anti-infective therapy in this population.

\section{Introduction}

Of the 7.1 million newly diagnosed tuberculosis (TB) cases notified to World Health Organization (WHO) in $2019,16 \%$ were EPTB ${ }^{1}$. Extrapulmonary tuberculosis (EPTB) can affect almost all organs and produce a wide range of clinical manifestations which poses challenges to effective management ${ }^{2}$. Epidemiological studies recently indicate that the proportion of EPTB could be increasing all over the world including China ${ }^{3-5}$. According to the WHO, when EPTB combined with pulmonary tuberculosis (PTB), it is usually reported as PTB ${ }^{6}$. Moreover, the diagnosis of EPTB is clinically challenging, so the actual incidence of EPTB may be even higher ${ }^{6}$.

Despite significant national achievements in addressing the TB epidemic, China still faces a high burden of RR囚MDR-TB ${ }^{7}$.According to the WHO, only $54 \%$ of the MDR-TB patients successfully complete treatment ${ }^{8}$. However, to our knowledge, clinical and demographic descriptions of RR/MDR-EPTB are still lacking. In recent years, highly effective molecular etiology techniques have been widely used in clinical practice ${ }^{9}$. With the wide range applications of Gene Xpert MTB/RIF区Xpert\assay in China and increasing national regulatory concern on RR/MDR-TB, RR/MDR-EPTB has also increasingly attracted the attention of goverment and researchers. Considering the multiple organ-specific clinical symptoms and non-specific 
systemic symptoms, the in-depth understanding of the clinical characteristics of RR/MDR-EPTB is important in order to develop appropriate protocols for management. Therefore, the clinical data collected from a national TB clinical research center in China sicene 2015 to 2019 were reviewed to summarize the clinical characteristics of RR/MDR-EPTB \and compared with RR/MDR-PTB at the same period. To our knowledge, this is the first time that data on RR/MDR-EPTB have been collected since the Xpert widely introduced in China from 2015. Our aim was to observe the demographic and clinical characteristics of RR/MDR-EPTB $\square$ its occurrence in different organs $\square$ the characteristics of the drug resistance spectrum $\square$ and its differences from RR/MDR-PTB, for better prevention and control of the disease.

\section{Materials And Methods}

\section{Ethics statement}

The retrospective study was conducted at a national TB clinical research center with nearly 300 beds that received the referrals for RR/MDR-TB patients from east China. We also undertakes the management of the regional RR/MDR-TB patients in cooperation with the Shanghai Municipal Center for Disease Control and Prevention (CDC). The retrospective study was approved by The Ethics Committee of Shanghai Pulmonary Hospital (approval number k17-138).

\section{Data Sources and Collection:}

The electronic medical record system recorded the patient's demographic characteristics and clinical data. All patients included were regularly followed up in thehospital and the records were complete. A total of 1553 RR/MDR-TB patients were recorded in the electronic database of Shanghai pulmonary hospital from January 2015 to December 2019. After excluding 43 patients with incomplete clinical datas, 1510 patients were included in the final statistics (fig1). We collected multiple demographic and clinical variables from electronic records. The clinical data of different types of RR/MDR-EPTB group and RR/MDR-PTB group were extracted and compared. The data included sex $\square$ age $\llbracket$ residence $\llbracket p r e v i o u s$ TB history $\square$ complications with diabetes $\square$ microbiological data $\llbracket$ treatment outcomes, etc.

\section{Definition and Inclusion Criterial}

Cases were classified according to the main anatomical site. The PTB group was defined as the lung being the only pathogenic anatomical site. The EPTB group was defined as tuberculous infection at any anatomical site except the lung, regardless of the presence of PTB ${ }^{10,11}$.

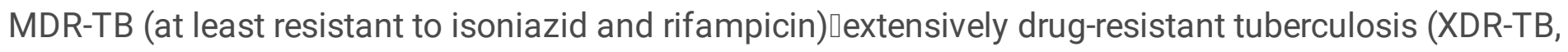
MDR-TB resistance to both fluoroquinolones and second-line injection agents) or pre-XDR (MDR-TB resistant to either fluoroquinolones or second-line injection agents) were defined through drug sensitivity test (DST) based on mycobacterium tuberculosis (MTB) culture ${ }^{12}$. 
Resistance to Rifampicin is mainly associated with mutations in a limited region of the $r p o b$ gene ${ }^{13}$, which may occur alone or may be associated with other drug resistance. In high MDR-TB settings, the presence of resistance to rifampicin alone can be considered MDR-TB ${ }^{14}$. In the present study, resistance to rifampicin was determined based on MTB culture DST or genotype DST (Xpert). Shanghai CDC requires all patients suspected TB to be tested for Xpert when newly diagnosed, as well as MTB culture. In accordance with the recommendations of the Global Laboratory Initiative (GLI) and the World Health Organization ${ }^{15}$, TB patients who are not at high risk of drug resistance should undergo two times of Xpert tests to determine the presence of drug resistance. Thus, In the present study, a second Xpert test was performed on newly diagnosed patients .

Inclusion criteria: 1) MTB culture was positive, and MTB cultural-based DST indicated at least rifampicin resistance on extrapulmonary specimens. 2) Xpert MTB positive and rifampicin resistance detected on extrapulmonary specimens. 3) Complete clinical datas and regular follow-up.

The delay in MTB detection is defined as the time (days) between the first visit to hospital and the date when RR/MDR-EPTB was confirmed ${ }^{16}$.

We defined newly diagnosed MDR-TB as having never received ATT treatment or less than one month of treatment ;Retreatment cases were defined as registered MDR-TB patients who had previously taken firstline anti-TB drugs for more than one month; or with evidence of taking first-line anti-TB drugs in the past month or longer ${ }^{17}$.

\section{Microbiological assessment}

Appropriate samples are taken from the anatomical site and sent to a microbiology laboratory. Body fluid /tissue samples were collected for Xpert and MTB culture. BACTECTM MGIT 960 culture (MGIT 960) was used to conduct the MTB culture and DST. MGIT960 system, MGIT culture tube and SIRE kit were purchased from BD company from United States .

According to the instructions of the Gene Xpert MTB/RIF (Xpert) testing system (Cepheid, USA, Version 4), homogenate tissues were mixed with GeneXpert reagent, incubated at room temperature for a short period, and then transferred to the cartridge and inserted into GeneXpert device. The test results were automatically interpreted after 2 hours .

\section{Treatment regimens and follow-up]}

Treatment regimens were individualized according to WHO guidelines and DST results (18). All enrolled patients were regularly treated and followed-up, and direct observation therapy (DOT) was used throughout the treatment process.

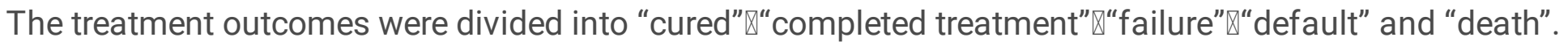
Treatment outcome datas were collected 18 months after start of treatment. The favorable treatment outcomes were defined as sum of "cured" and "treatment completed" (19). 


\section{Statistical analysis}

Intergroup differences (PTB and EPTB; Different anatomical site of EPTB) were determined by the chisquare test or Fisher's exact test to obtain significant factors. We tabulated the demographic and clinical data of the cases and analyzed the relevant factors using logistic regression models. Odds ratios (ORs) with $95 \%$ confidence intervals (Cls) between groups were calculated. If $P<0.01$, the difference was considered statistically significant. Statistical analysis was performed using Stata 14 software (Statacorp, Texas, United States (US).

\section{Results}

\section{Study population and distribution of extrapulmonary anatomical site $\square$}

Among all patients enrolled $(n=1510), 84.6 \% \bigotimes 1278 / 1510 \rrbracket$ were PTB and $15.4 \% \varangle 232 / 1510 \rrbracket$ were EPTB. The most common anatomical site was pleural/chest wall $(25.9 \% \varangle 60 / 232)$. Additional

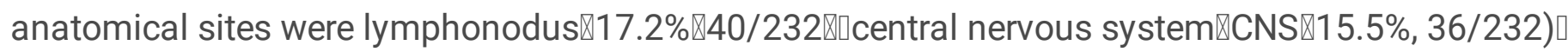

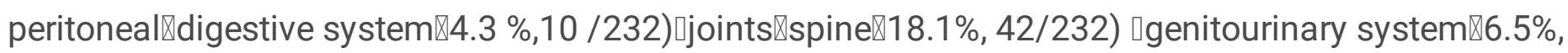

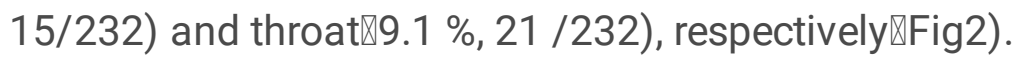

\section{Demographic analysis and clinical characteristics of RR/MDR-EPTB compared RR/MDR-PTB:}

We summarized the demographic and clinical characteristics of patients with RR/MDR-EPTB versus $\llbracket v$ s $\llbracket$ RR/MDR-PTB (Table1). Although the proportion of males was higher than females in both RR/MDR-PTB $\triangle 72 \%$ vs $28 \%$ and RR $\square$ MDR-EPTB $₫ 53.5$ vs $46.5 \%$, relatively more females were likely to be susceptible to RR/MDR-EPTB (OR 1.65, 95\% Cl 1.52-1.77). The distribution of RR/MDR-EPTB was also different in different age groups. Using the caess of the age $<25$ years as the control group, we found that the risk of RR/MDR-EPTB (25-44ðyears group increased (OR 1.61, 95\% Cl 1.52-1.77), and then decreased with the increasing age (OR1.48 95\% $\mathrm{Cl}$ 0.74-1.69 for 44-65 years group and OR 2.23 95\% $\mathrm{Cl}$ 0.98-2.71 for $\geq 65$ years group). Patients in rural areas seemed to have an increased risk of both RR囚MDR-PTB and RR/MDR -EPTB compared with patients in urban areas (OR $0.63,95 \% \mathrm{Cl} 0.51-1.21$ ).

In addition, patients with RR/MDR-EPTB were more likely to be newly diagnosed than those with RR/MDRPTB (OR 1.22ه95\% $\mathrm{Cl} 1.21-2.05)$. Compared with RR/MDR-EPTB, patients with diabetes were more likely to develop RR/MDR-PTB (OR 1.45, 95\% Cl 1.04-1.87区.

In terms of etiology, RR/MDR-EPTB has a significant delay in diagnosis compared with RR/MDR-PTB

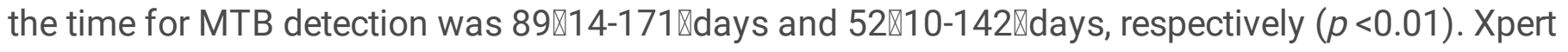
obtained similarly high detection rates in RR/MDR-PTB and RR/MDR-EPTB ( $p 0.24)$, and culture detection rates in RR/MDR-PTB were significantly higher than in RR/MDR-EPTB $(p<0.01)$.Notably, we found significantly higher rates of pre-XDR in RR/MDR-EPTB $(p<0.01)$.

\section{Clinical characteristics of different anatomical sites of RR/MDR-EPTBD}


In the study, women were more likely than men to have tuberculous lymphadenitis (OR $1.4,95 \% \mathrm{Cl} 1.0-1.8$ ) and genitourinary TB $(\mathrm{OR} 2.7,95 \% \mathrm{Cl}$ 1.3-3.1) ; while men were more likely than women to develop pleurisy凶chest wall TB (OR 1.4, 95\% Cl 0.6-1.8) पperitoneal『digestive system TB (OR 1.5, 95\% Cl 1.6-3.8) $\square$ CNS TB (OR 1.27, 95\% Cl 0.7-2.8) djoints \spine TB (OR 1.5, 95\% Cl 1.2-3.9) and throat TB (OR 1.3, 95\% Cl 1.2-2.9). Pleural/chest wall TB tended to be relatively young with the median age of 25.6 (15-58) years old while CNS TB tended to be relatively old with the median age of 49.1(15-72ðyears old. Patients from urban were more likely to develop peritonealखdigestive system TB than those from rural (OR $2.33,95 \% \mathrm{Cl}$ 0.56-2.78). Other types of RR/MDR-EPTB were mainly from rural areas. In terms of the TB history, tuberculous lymphadenitis (OR 1.3, 95\% Cl 1.3-2.1) \pleural /chest wall TB (OR 1.6, 95\% Cl 1.7-2.8) $\square \mathrm{CNS}$ TB (OR 1.6, 95\% $\mathrm{Cl} 1.4-2.2)$ and genitourinary system TB (OR 1.6, 95\% $\mathrm{Cl} 2.4-3.9)$ were more likely to be newly diagnosed $\llbracket$ while most of joint /spinal TB and throat TB were re-treated patients. The longer delay

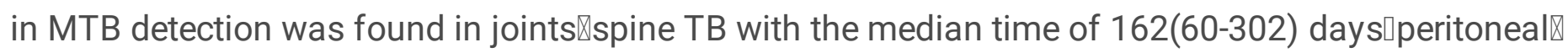
digestivesystem TB with the median time of 128 (14-160) days $\square$ genitourinary system TB with the median time of 105 (20-126) days and pleural/chest wall TB with the median time of 101 (21-192). (Table 2 )

\section{Total number of RR/MDR-EPTB over the study period:}

We further analyzed the the total number of RR/MDR-EPTB over the study period (Figure 3a). During 2015-2019, the proportion of RR \MDR-EPTB in total cases of RR/MDR-TB has increased from 8.8 $\% \otimes 14 / 160 \rrbracket$ to $18.1 \% \varangle 66 / 364 \rrbracket \square P<0.01$ hhowever, the number of RR/MDR-EPTB cases increased from 14 cases $(6.0 \%, 14 / 232)$ in 2015 to 66 cases $(28.4 \%, 66 / 232)$ in 2019. Analysis of RR/MDR-EPTB at different anatomical sites found that: the number of spinal『joint TB and CNS TB seen to be increasing from 2015-2019凶the number of tuberculous lymphadenitis $\square$ pleural /chest wall TB and peritoneal $\llbracket$ digestive system TB was increasing from 2015-2018 but declined in 2019.

\section{Drug resistance characteristics of RR/MDR-EPTBD}

The RR/MDR-EPTB was diagnosed mainly based on Xpert $(90.9 \%, 211 / 232)$ rather than the MTB culture $(56.0 \%, 130 / 232)$.We found $70(30.2 \%, 70 / 232)$ cases of pre-XDR/XDR-EPTB, which was significantly higher than that in MDR/RR-PTB $₫ 18.3 \%, 234 / 1278 \llbracket$ during the same period. The pre-XDR/XDR-EPTB was more common in joints $₫$ spine $T B \llbracket 40.5 \%, 17 / 42 \rrbracket$ and tuberculous lymphadenitis $₫ 31.2 \% \bigotimes 15 / 232 \rrbracket$. Resistance to ethambutol $₫ 74.6 \% \bigotimes 173 / 232$ and streptomycin $₫ 87.5 \% \varangle 203 / 232 \rrbracket$ were common while resistance to cycloserine $(5.6 \%, 13 / 232)$ nethionamide $(12.9 \%, 30 / 232)$ and paminosalicylic acid $(8.2 \%, 19 / 302)$ were relatively rare. Rifabutin was sensitive to some MDR/RR-EPTB patients $(12.5 \%$, 29/232).

\section{Treatment outcome:}

All 232 patients received treatment for at least 18 months. The favorable treatment outcomes at the 18 months was reported in $61.2 \%$ (142/232) of RR/MDR-EPTB, significantly lower than that of the RR/MDRPTB $(70.7 \%, 903 / 1278)$ in the study period, $p<0.01$. Among them, the favorable treatment rate was 
relatively lower in joints/spine TB $(53.3 \%, 8 / 15)$ and genitourinary TB $(52.4 \% \bigotimes 22 / 42)$ but higher in throat $\operatorname{TB}(76.2 \%, 16 / 21)$.

A total of 5 patients of MDR-EPTB died during the study period as a direct result of TB囚2 were CNS TB 1 was pleural TB complicated with tuberculous empyema and bronchopleural fistula 1 was digestive system TB complicated with intestinal perforation and acute peritonitis and 1 was cervical tuberculosis complicated with paraplegia. Additionally, 11 RR/MDR-PTB patients died during the study period, the direct causes of death were 6 of respiratory failure due to multiple infections , 3 of cardiac failure $\mathbb{1}$ of massive hemoptysis and 1 of diabetic ketoacidosis.

\section{Discussion}

China has a high incidence of TB and drug-resistant TB worldwide ${ }^{1}$. Limited epidemiological information suggests that the incidence of EPTB in China may be increasing ${ }^{3,4}$. However, there is still a lack of large sample data to summarize the clinical information of RR囚MDR-EPTB populations worldwide. In the present study, we included 5 years of data on all well-documented MDR-TB , in which $17.8 \%$ were EPTB and the most common site were pleura/chest wall (25.9\%) and lymphonodus (20.9\%). We also estimated the incidence trend of RR/MDR-EPTB over the 5 years period through looking at the number of cases. We found that the proportion of RR/MDR-EPTB in total MDR-RR/TB cases increased from $8.8 \%$ 14/160冈to $18.1 \% \varangle 66 / 364 \mathbb{} \otimes P<0.01$; the number of RR/MDR-EPTB cases increased from 14 cases $(6.0 \%, 14 / 232)$ in 2015 to 66 cases $(28.4 \%, 66 / 232)$ in 2019. As we collected data from TB specialist hospitals that received referrals, it may not be representative of the epidemic in the region. The increasing trend may be due to the the introduction of molecular MTB detection techniques for the management of MDR-TB in China. Consistent with existing studies on EPTB ${ }^{4,11}$, females were more likely to be susceptible to RR/MDR-EPTB (OR 1.65, 95\% Cl 1.52-1.77) \more likely to be newly diagnosed $₫ p<0.01$ \and less likely to to combine with diabetes $₫ P<0.01)$. Notably, even with the introduction of highly sensitive molecular detection techniques, RR/ MDR-EPTB was still delayed in detection, with a median time of 89(14-171) days from the first visit. This makes sense, given the diagnostic challenges of EPTB, the difficulty of obtaining microbiological evidence in the early stages of the disease, and the fact that some require invasive biopsies or even surgery. Moreover, most of the extrapulmonary specimens lack viable bacteria, making the traditional MTB culture method extremely low in positive rates and requiring weeks to obtain results ${ }^{6}$. We also found that, RR/MDR-EPTB relied more on Xpert to establish the diagnosis. In the study, Xpert was positive in $89.9 \%$ cases of RR/MDR-PTB and $90.9 \%$ cases of RR/MDR-EPTB, while the culture positive rate of MDR/RR-EPTB was significantly lower than that of RR/MDR-PTB. ( $56 \%$ vs $70 \%, P<0.01$ ). Previous study shown that Xpert should be used as a primary diagnostic tool for the detection of EPTB, especially in tuberculous lymphadeniti, However its added-value in diagnosing pleural or peritoneal TB may be limited ${ }^{20}$. Existing studies, however, have focused on the microbiological diagnosis of EPTB, rather than on drug-resistant populations. Effective treatment of RR/MDR-TB is based on accurate detection of drug resistance, but microbiological data or related studies on RR/MDR-EPTB were still lacking. Delay in MTB detection was likely to be associated with an increase in bacterial load and 
progression of the disease. Inaccurate treatment may acquire more resistance, leading to further spread of the disease ${ }^{21}$. Rapid molecular diagnostic tests such as Xpert significantly reduce the time in MTB detection, and thus the time to initiate the correct treatment, compared to traditional DST ${ }^{22}$. In our study, we found that Xpert could detect most RR/MDR-EPTB and had good performance in most kinds of specimens. A large study from China showed that the etiological positive rate of EPTB was low (12.8\%) ${ }^{4}$. Existing review concluded that the sensitivity of Xpert pooling (defined by culture) in EPTB varies between specimen types (31\% in pleural tissue and $97 \%$ in bone or articular fluid); Sensitivity of Xpert in urine, bone joint fluid, and tissue is $>80 \%$. Meanwhile, in RR- EPTB, Xpert pooled sensitivity and specificity were $95.0 \%$ and $98.7 \%$, respectively ${ }^{23}$.However, non-drug resistant EPTB cases were not included in the study, so no specific discussion was carried out. The purpose of this point is to illustrate the value of Xpert in the rapid diagnosis of RR/EPTB. We hypothesize that the processes leading to drug resistance or the infection of resistant bacteria may increase the local bacterial load.

Fluoroquinolones $\triangle \mathrm{FQ}$ s $₫$ have long been considered the cornerstone of treatment regimens for MDR$\mathrm{TB}^{24}$ and listed as Group A recommended drugs in the latest guidelines ${ }^{18}$. Increased second-line drug resistance was associated with a higher rate of treatment failure ${ }^{25}$. However, FQS has been widely used to treat bacterial infections of respiratory tract $\square$ gastrointestinal tract and urinary tract in China over the past decades ${ }^{26}$. Improper use of FQs may lead to acquired resistance in TB patients, which in turn leads to the spread of the strains that have already resistant to FQs. Molecular epidemiological study also shown that resistance to moxifloxacin increased significantly in China between 2000 and $2010^{27}$. In our study, we also found $30.2 \%$ cases of pre-XDR/XDR-EPTB, which was significantly higher than that in MDR/RR-PTB $\nabla 18.3 \%, P<0.01 \rrbracket$, especially in joint $\llbracket$ spinal $T B \otimes 40.5 \% \bigotimes$. The probable reason is that the patients may have been using FQs as empiric anti-infective therapy for a longer period of time before being diagnosed with TB. Our results also revealed that the majority of RR / MDR-EPTB were newly diagnosed. Because the early manifestations of EPTB are indistinguishable from common bacteria infection (genitourinary infection $\square$ bone and joint infection, etc.), patients may often first referred to a non-TB specialist hospitals, so antibiotics may be administered while waiting for definition. In the present study, the treatment success rate after 18 months was $70.7 \%$ in MDR/RR-EPTB , significantly lower than that of RR/MDR-PTB ( 61.2 $\%)$ in the same period. Among them, the favorable treatment rate was lower in joint/spinal TB (52.4\%) and genitourinary TB (53.3\%) . Our results also revealed that the prognosis of RR/MDR-EPTB may be worse than that of RR/MDR-PTB, and FQS resistance may be one of the main reasons. Clinicians should use Xpert for rapid diagnosis as early as possible in patients with suspected TB and avoid using FQs for routine anti-infective therapy.

Our study had some limitations. EPTB was not included in routine infectious disease reports in China CDC due to its relatively low infectivity. Our retrospective study only collected datas from a TB/pulmonary disease specialized hospital. There was a selection bias at the time of inclusion and was not representative of regional prevalence. In addition, when we observed RR/MDR-EPTB, we did not include non-drug-resistant EPTB synchronously for comparison, which may not directly reflect the characteristics 
of this disease in some aspects. However, our findings highlight the need for rigorous management and monitoring of RR/MDR-EPTB.

\section{Conclusion}

Our study identified three main concerns for RR/MDR-EPTB. Firstly, most types of RR/MDR-EPTB detected in the hospital could been on the rise in recent years, which may be partly due to the progress of detection technology. Secondly, RR/MDR-EPTB may be diagnosed with a delay that is longer than RR/MDR-PTB. Xpert should be recommended for all patients suspected EPTB, especially in areas lack of laboratory conditions for MTB culture. Thirdly, higher rate of resistance to FQs in RR囚MDR- EPTB may be associated with a worse prognosis, thus clinicians should use FQs cautiously in cases suspected TB and pay close attention to FQs DST when developing treatment region. These issues should be addressed in TB surveillance and control programme implemented.

\section{Declarations}

\section{Ethics approval and consent to participates:}

The study methodologies conformed to the standards set by the Declaration of Helsinki. The retrospective study was approved by The Ethics Committee of Shanghai Pulmonary Hospital (Approval number K17138). The informed consent was obtained for all participants before inclusion of clinical datas and biological sample datas.

\section{Consent for publication:}

Not applicable

\section{Availability of data and materials:}

All datas regarding the included participants and laboratory data during the study are available from the corresponding author by email request. The study followed the CONSORT guidelines.

\section{Competing interests:}

The authors declare that they have no competing interests.

\section{Funding acknowledgements:}

This work was supported by clinical cultivation project of Shanghai pulmonary hospital (FKLY20017) and Shanghai science and techonlogy commettee fund (21Y11901000). The funders had no role in study design, data collection and analysis, decision to publish, or preparation of the manuscript.

\section{Author contribution Statement:}


All authors made a significant contribution to the work reported, whether that is in the conception, study design, execution, acquisition of data, analysis and interpretation, or in all these areas; took part in drafting, revising or critically reviewing the article; gave final approval of the version to be published; have agreed on the journal to which the article has been submitted; and agree to be accountable for all aspects of the work.

\section{Acknowledgements:}

We thank all participants for their time and efforts.

\section{References}

1 WHO. Global tuberculosis report 2020. Geneva: World Health Organization; 2020.

2 Solovic I, Jonsson J, Korzeniewska-Koseła M, Chiotan DI, Pace-Asciak A, Slump E, et al. Challenges in diagnosing extrapulmonary tuberculosis in the European Union, 2011. Euro Surveill. 2013;18:20432

3 Wang X, Yang Z, Fu Y, Zhang G, Wang X, Zhang Y, et al. Insight to the epidemiology and risk factors of extrapulmonary tuberculosis in Tianjin, China during 2006-2011. PLoS One. 2014;9:e112213.

4 Pang Yu, An Jun, Shu Wei et al. Epidemiology of Extrapulmonary Tuberculosis among Inpatients, China, 2008-2017 .Emerging Infect. Dis., 2019, 25: 457-464.

5 Sandgren A, Hollo V, van der Werf MJ. Extrapulmonary tuberculosis in the European Union and European Economic Area, 2002 to 2011. Euro Surveill. 2013;18:20431.

6 Kohli M, Schiller I, Dendukuri N, Yao M, Dheda K, Denkinger CM, Schumacher SG, Steingart KR. Xpert MTB/RIF Ultra and Xpert MTB/RIF assays for extrapulmonary tuberculosis and rifampicin resistance in adults. Cochrane Database of Systematic Reviews 2021, Issue 1.

7 Huo F, Luo J, Shi J, Zong Z, Jing W, Dong W, et al. A 10-year comparative analysis shows that increasing prevalence of Rifampin-resistant Mycobacterium tuberculosis in China is associated with the transmission of strains harboring compensatory mutations. Antimicrob Agents Chemother. 2018; 62 (4) :e02303-e2317

8.World Health Organization. Global Tuberculosis Report. Geneva, World Health Organization, 2017.

9 Keikha M, Karbalaei M. High resolution melting assay as a reliable method for diagnosing drug-resistant TB cases: a systematic review and meta-analysis. BMC Infect Dis. 2021;21(1):989.

10 Hayward S E, Rustage K, Nellums L B , et al. Extrapulmonary tuberculosis among migrants in Europe, 1995 to 2017 - ScienceDirect . Clinical Microbiology and Infection, 2020. 
11 Peto HM, Pratt RH, Harrington TA, LoBue PA, Armstrong LR. Epidemiology of extrapulmonary tuberculosis in the United States, 1993-2006. Clin Infect Dis. 2009;49:1350-7

12 World Health Organization (WHO). Extensively DR tuberculosis (XDR.TB): recommendations for prevention and control. Wkly Epidemiol Rec. 2006;81:430-2.

13 Telenti A, Imboden P, Marchesi F, Lowrie D, Cole S, Colston MJ, et al.Detection of rifampicin-resistance mutations in Mycobacterium tuberculosis . Lancet 1993;341(8846):647-50.

14 World Health Organization. Global tuberculosis report 2018. Geneva, Switzerland: World Health Organization, 2018.

15 GLI model TB diagnostic algorithms. Global Laboratory Initiative; 2017. http:// www. stoptb. org/ $\mathrm{wg} / \mathrm{gli}$ / assets/ documents / GLI_ algorithms.pdf.

16 Wu Zheyuan,Rueda Zulma Vanessa,Li Tao et al. Effect of the Xpert MTB/RIF on the detection of pulmonary tuberculosis cases and rifampicin resistance in Shanghai, China .BMC Infect Dis, 2020, 20: 153.

17 Sun W, Wu Z, Zhou Y, et al. A highly effective and inexpensive standardized treatment of multidrugresistant tuberculosis: a multicenter prospective study in China. BMC Infect Dis. 2021;21(1):834.

15WHO. WHO consolidated guidelines on drug-resistant tuberculosis treatment. world health organization. $2019 ; 2019$.

19 Duan H, Chen X, Li Z, Pang Y, Jing W, Liu P, et al. Clofazimine improves clinical outcomes in multidrugresistant tuberculosis: a randomized controlled trial. Clinical microbiology and infection : the official publication of the European Society of Clinical Microbiology and Infectious Diseases. 2019;25(2):190-5.

20 Tadesse M,Abebe G,Bekele A et al. Xpert MTB/RIF assay for the diagnosis of extrapulmonary tuberculosis: a diagnostic evaluation study .Clin Microbiol Infect, 2019, 25: 1000- 1005.

21 Yang C, Luo T, Shen X, Wu J, Gan M, Xu P, Wu Z, Lin S, Tian J, Liu Q, Yuan Z, Mei J, DeRiemer K, Gao Q. 2017. Transmission of multidrug-resistant Mycobacterium tuberculosis in Shanghai, China: a retrospective observational study using whole-genome sequencing and epidemiological investigation. Lancet Infect Dis 17:275-284.

22 Boehme CC, Nicol MP, Nabeta P, Michael JS, Gotuzzo E, Tahirli R, Gler MT, Blakemore R, Worodria W, Gray C, Huang L, Caceres T, Mehdiyev R, Raymond L, Whitelaw A, Sagadevan K, Alexander H, Albert H, Cobelens F, Cox H, Alland D, Perkins MD. 2011. Feasibility, diagnostic accuracy, and effectiveness of decentralised use of the Xpert MTB/RIF test for diagnosis of tuberculosis and multidrug resistance: a multicentre implementation study. Lancet 377:1495-1505. 
23 Kohli M, Schiller I, Dendukuri N, et al. Xpert® MTB/RIF assay for extrapulmonary tuberculosis and rifampicin resistance. Cochrane Database Syst Rev.2018;8(8):CD012768.

24 Collaborative Group for the Meta-Analysis of Individual Patient Data in MDR-TB treatment2017,Ahmad Nafees,Ahuja Shama D et al. Treatment correlates of successful outcomes in pulmonary multidrug-resistant tuberculosis: an individual patient data meta-analysis.Lancet, 2018, 392: 821-834.

25 D. Falzon, N. Gandhi, G.B. Migliori, G. Sotgiu, H.S. Cox, T.H. Holtz, et al. Resistance to fluoroquinolones and second-line injectable drugs: impact on multidrug-resistant TB outcomes. Eur Respir J, 42 (1) (2012), pp. $156-168$

26 Z. Zhang, J. Lu, Y. Wang, Y. Pang, Y. Zhao.Prevalence and molecular characterization of fluoroquinolone-resistant Mycobacterium tuberculosis isolates in China.Antimicrob Agents Chemother, 58 (1) (2014), pp. 364-369

27 Y. Pang, J. Lu, F. Huo, Y. Ma, L. Zhao, Y. Li, et al.Prevalence and treatment outcome of extensively drugresistant tuberculosis plus additional drug resistance from the national clinical center for tuberculosis in china: a five-year review. J Infect (2017), Article S0163445317302621.

\section{Tables}

Table 1: Comparison of clinical characteristics of RR/MDR-PTB Vs RR/MDR-EPTB during the study period, 2015-2019: 
RR/MDR-PTB

$(\mathrm{n}=1278)$
RR/MDR-EPTB

$(n=232)$
Odds ratio

(95\% CI)

Sex $(n, \%)$

Male

Female

Age group (years )

$$
<25
$$

25-44

45-64

$\geq 65$

Residence (n,\%)

Rural

Urban

TB History (n,\%)

newly

Retreated

Diabetes (n,\%)

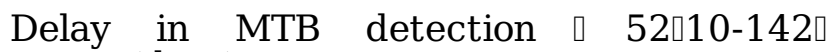
range (days)

Xpert positive (n,\%)

Culture positive $(\mathrm{n}, \%)$

XDR /pre-XDR (n,\%)

Favorable treatment outcomes $(\mathrm{n}, \%)$
$920 \square 72.0 \square$

$358 \square 28.0 \square$

$196 \square 15.3 \square$

$424 \square 33.2 \square$

$476 \square 37.2 \square$

$182 \square 14.2 \square$

$830 \square 64.9 \square$

$448 \square 35.1 \square$

$391 \square 30.6 \square$

887ロ69.4ロ

$479 \square 37.4 \square$

$1149 \square 89.9 \square$

$894 \square 70.0 \square$

$234 \square 18.3 \square$

$903 \square 70.7 \square$
$124(53.5)$

108 (46.5)

$39 \square 16.8 \square$

$124 \square 53.4 \square$

$54 \llbracket 23.3 \square$

$15 \square 6.5 \square$

$140(60.3 \square$

$92 \square 39.7 \square$

$110 \square 47.4 \square$

122 ( 52.6)

$59 \square 25.4 \square$

89๑14-171๘

211 ( 90.9)

130 (56.0)

$70(30.2)$

$142 \llbracket 61.2 \square$
$1.65 \quad(1.52-\quad *$
$1.77)$
Reference

Reference

$\begin{array}{lll}1.61 & 0.87- & * \\ 2.53 \square & & <0.01 \\ 1.48 & (0.74- & * \\ 1.69) & & <0.01 \\ 2.23 & 0.98- & * \\ 2.71 & & <0.01\end{array}$

$0.63 \quad(0.51-\quad 1.58$

1.21)

Reference

*:The difference was statistically significant

Table2₫Clinical characteristics of RR/MDR-EPTB in different sites : 


\begin{tabular}{|c|c|c|c|c|c|c|c|}
\hline Variable & $\begin{array}{l}\text { lymphonodus } \\
\square \mathrm{n}=48 \square\end{array}$ & $\begin{array}{l}\text { pleural } \square \text { chest- } \\
\text { wall } \\
(\mathrm{n}=60 \square\end{array}$ & $\begin{array}{l}\text { peritoneal } \square \text { digestive } \\
\text { system } \square \mathrm{n}=10 \square\end{array}$ & $\begin{array}{l}\text { CNS } \\
\square n=36 \square\end{array}$ & $\begin{array}{l}\text { Genitourinary } \\
\text { system } \llbracket n=15 \square\end{array}$ & joints $\square$ spine $\square n=42 \square$ & throat $\square \mathrm{n}=21 \square$ \\
\hline \multicolumn{8}{|l|}{ Sex $(n, \%)$} \\
\hline M & $20 \square 41.7 \square$ & $35 \square 58.3 \square$ & $4 \llbracket 40.0 \square$ & $20 \square 55.6 \square$ & $4(26.7 \square$ & $29 \square 69.0 \square$ & $12 \square 57.1 \square$ \\
\hline $\mathrm{F}$ & $28 \square 58.3 \square$ & $25 \llbracket 41.7 \square$ & $6 \square 60.0 \square$ & $16 \square 44.4 \square$ & $11(73.3 \square$ & $13 \llbracket 31.0 \square$ & $9 \llbracket 42.9 \square$ \\
\hline $\begin{array}{l}\text { Median age, years } \\
\text { (range) }\end{array}$ & $25.6(15-58)$ & $29.8(24-62)$ & $45.1(32-67 \square$ & $\begin{array}{l}49.1(15- \\
72 \square\end{array}$ & $45.7(29-71 \square$ & 43.3(19-68! & $48.6(16-71 \square$ \\
\hline \multicolumn{8}{|l|}{ Residence (n,\%) } \\
\hline Rural & $28 \square 58.3 \square$ & $34 \square 56.7 \square$ & $3 \square 30.0 \square$ & $22(61.1 \square$ & $10 \square 66.7 \square$ & $30 \square 71.4 \square$ & $13(61.9 \square$ \\
\hline Urban & 20ロ41.7ロ & $26 \llbracket 43.3 \square$ & $7 \square 70.0 \square$ & $14(38.9 \square$ & $5 \square 33.3 \square$ & $12 \llbracket 28.6 \square$ & 8(38.1] \\
\hline \multicolumn{8}{|l|}{ TB History $(\mathrm{n}, \%)$} \\
\hline newly & $26 \square 54.2 \square$ & $35 \square 58.3 \square$ & $5 \square 50.0 \square$ & $20 \square 55.6 \square$ & $9(60.0 \square$ & $10 \square 23.8 \square$ & $5 \square 23.8 \square$ \\
\hline Retreated & $22 \llbracket 45.8 \square$ & $25 \square 41.7 \square$ & $5 \square 50.0 \square$ & $16 \square 44.4 \square$ & $6 \square 40.0 \square$ & $32 \square 76.2 \square$ & $16 \square 76.2 \square$ \\
\hline Diabetes (n,\%) & $10 \square 21.7 \square$ & $14 \square 23.3 \square$ & $2 \llbracket 20.0 \square$ & $11 \square 30.6 \square$ & $4 \llbracket 26.7 \square$ & $11 \square 26.2 \square$ & 7ロ33.3ロ \\
\hline $\begin{array}{l}\text { Delay in MTB } \\
\text { detection } \square \text { Days (range) }\end{array}$ & $47(18-159)$ & $101(21-192)$ & $128(14-160)$ & $\begin{array}{l}31(18- \\
162)\end{array}$ & $105(20-126)$ & $162(60-302)$ & $32(14-82)$ \\
\hline Xpert positive (n,\%) & 45 (93.8) & 55 ( 91.7) & $8 \square 80.0 \square$ & $\begin{array}{l}32 \\
(88.9)\end{array}$ & $12(80.0)$ & 38 (90.5) & $21(100.0)$ \\
\hline Culture positive $(\mathrm{n}, \%)$ & $28(58.3)$ & $30(50.0)$ & $4(40.0)$ & $20(55.6)$ & $10(66.7)$ & $17(40.5)$ & $21(100.0)$ \\
\hline XDR /pre-XDR (n,\%) & $15(31.2)$ & $18(30.0)$ & $3(30.0)$ & $8(22.2)$ & $4 \llbracket 26.7 \square$ & $17(40.5)$ & $5 \square 23.8 \square$ \\
\hline $\begin{array}{l}\text { Favorable treatment } \\
\text { outcomes }(n, \%)\end{array}$ & $30 \square 62.5 \square$ & $36 \square 60 \square$ & $6 \square 60 \square$ & $24 \square 66.7 \square$ & $8 \square 53.3 \square$ & $22 \square 52.4 \square$ & $16 \square 76.2 \square$ \\
\hline
\end{tabular}

\section{Figures}

Figure 1

\section{Flow chart}




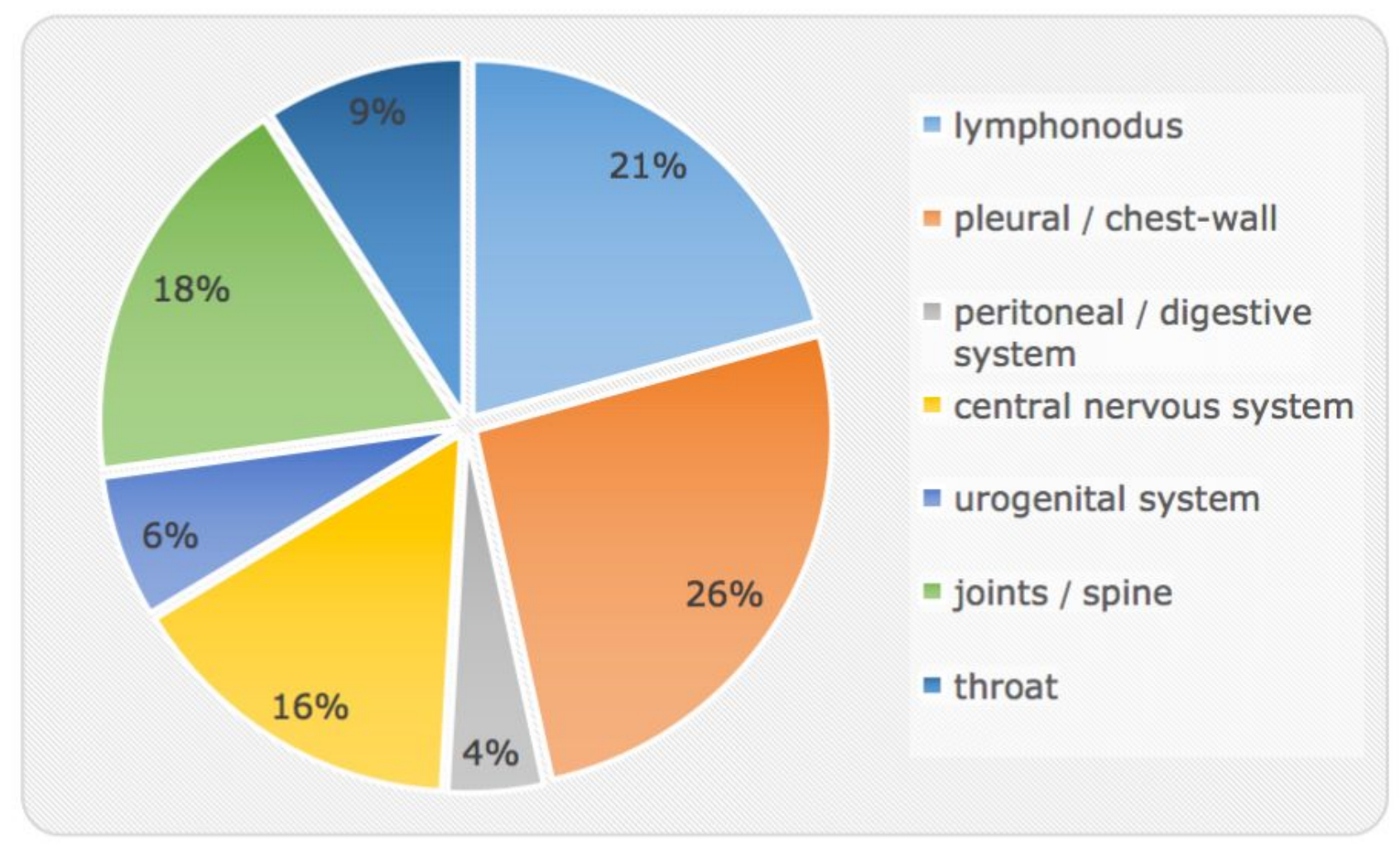

\section{Figure 2}

The anatomical site of RR/MDR-EPTB enrolled $\$

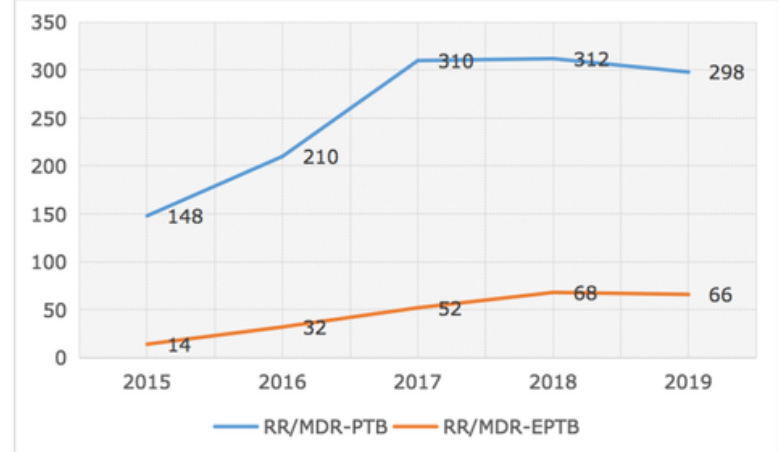

A

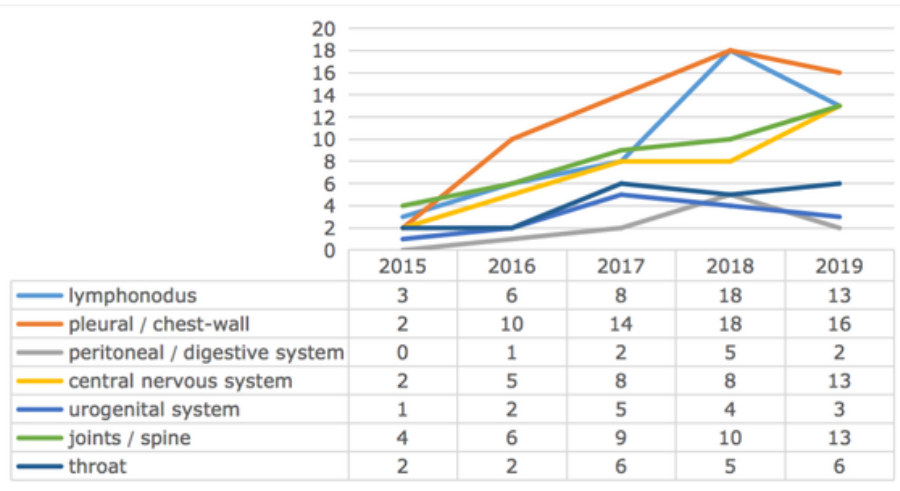

B

\section{Figure 3}

a Total number of RR/MDR-EPTB and RR/MDR-PTB in the study Period (n). b Total number of RR/MDREPTB at different sites during the study period $\llbracket n$ )

\section{Supplementary Files}


This is a list of supplementary files associated with this preprint. Click to download.

- GraphicalAbstract.png 